\title{
Developing a Robust, Interoperable GNSS Space Service Volume (SSV) for the Global Space User Community
}

Frank H. Bauer, FBauer Aerospace Consulting Services (FB-ACS)

Joel J. K. Parker, NASA Goddard Space Flight Center

Bryan Welch, NASA Glenn Research Center

Werner Enderle, ESA European Space Operations Center

ION International Technical Meeting

January 31,2017 


\section{Benefits of GPS/GNSS to NASA}

Real-time On-Board

Navigation: Enables new methods of spaceflight ops such as precision formation flying, rendezvous \& docking, station-keeping, GEO satellite servicing

Earth Sciences: GPS used as a remote sensing tool supports atmospheric and ionospheric sciences, geodesy, and geodynamics -from monitoring sea levels \& ice melt to measuring the gravity field

Attitude Determination: Use of GPS/GNSS enables some missions to meet their attitude determination requirements, such as ISS

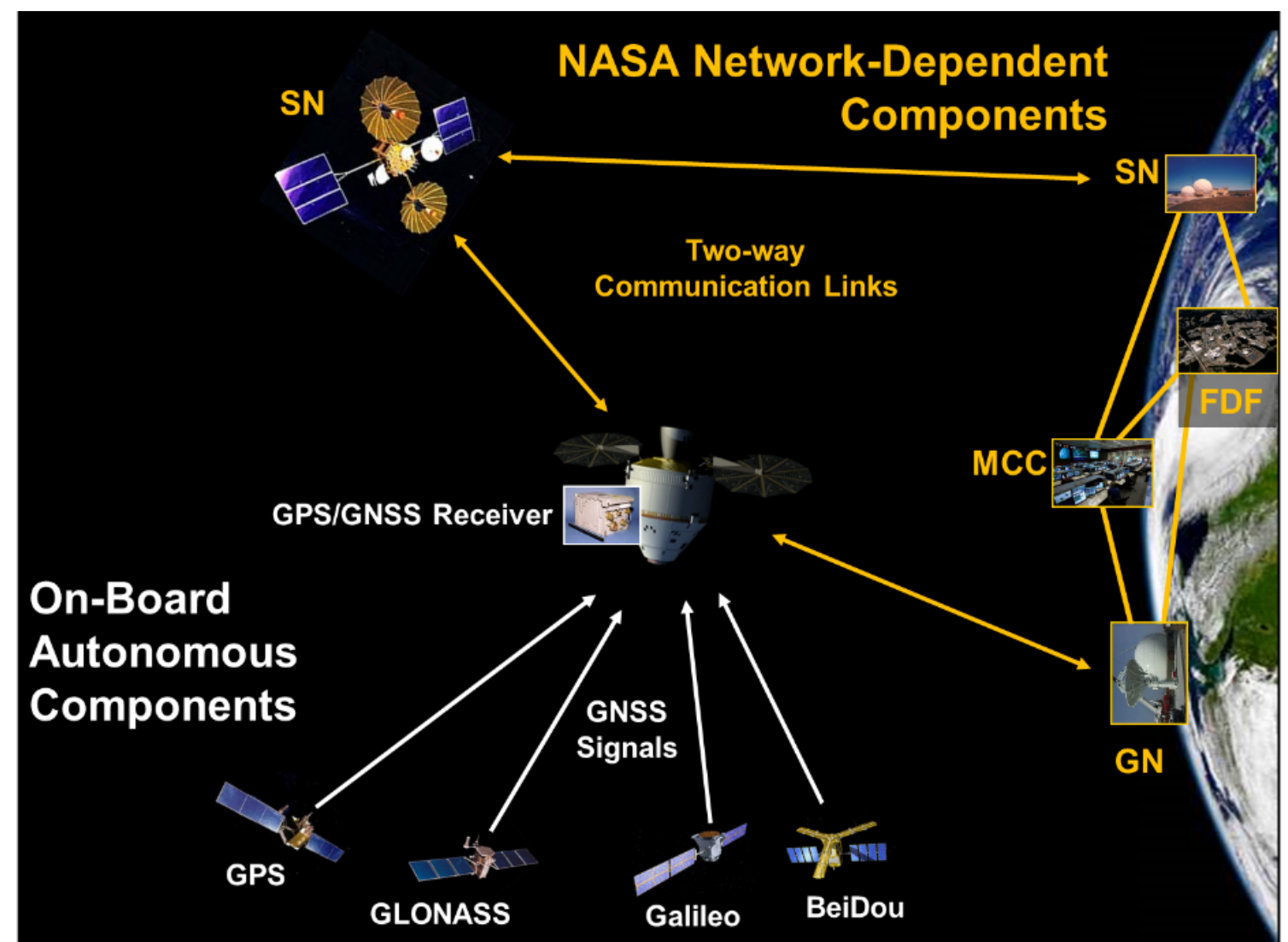

NASA is investing approximately $\$ 130 \mathrm{M}$ over the next 5 years on GPS R\&D and its implementation in support of space operations and science applications GPS capabilities to support space users may be further improved by pursuing compatibility and interoperability with GNSS (Global Navigation Satellite Systems), such as the Russian GLONASS, European Galileo, and China's BDS 
NASA Reception Geometry for GPS Signals in Space

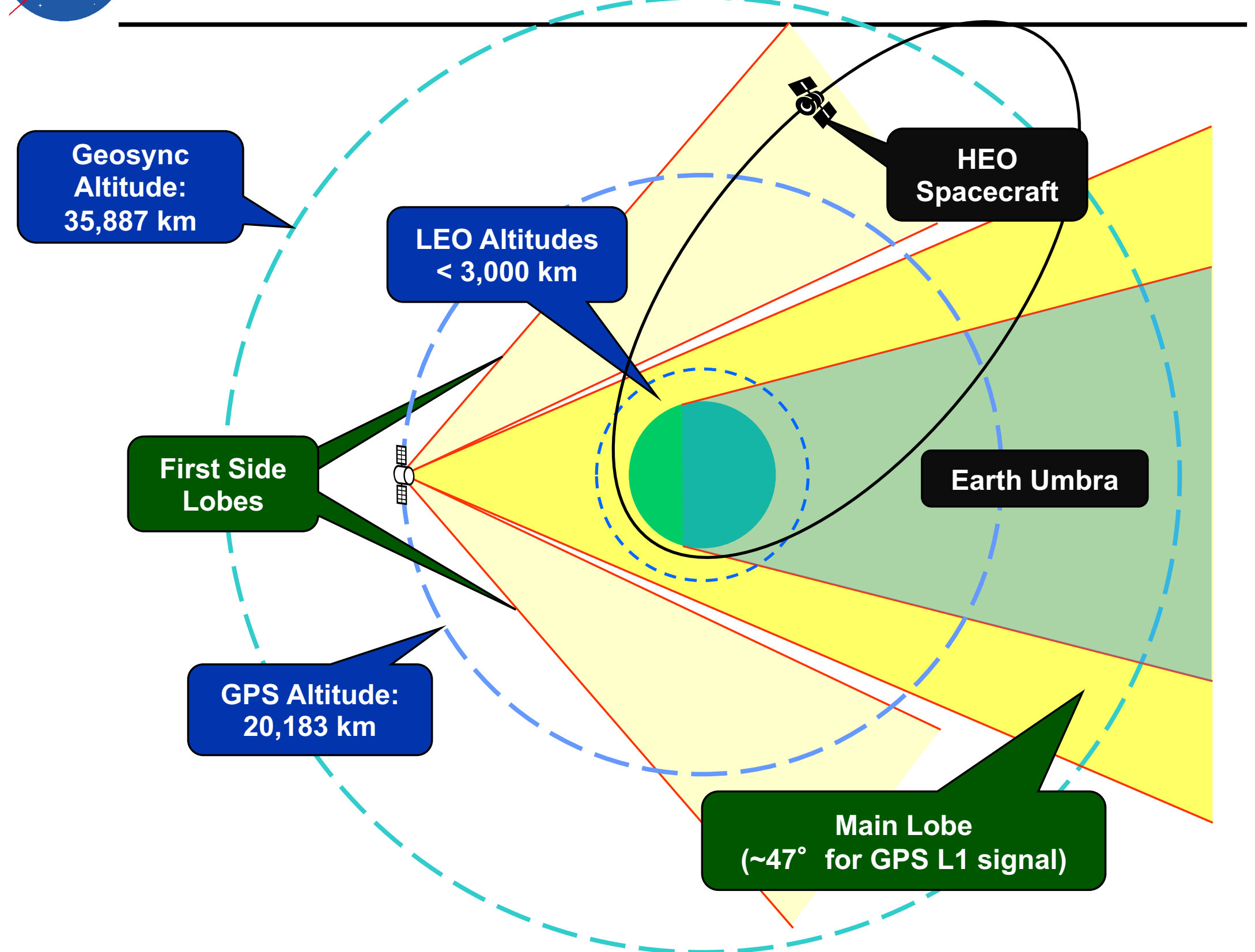




\section{What is a Space Service Volume (SSV)?}

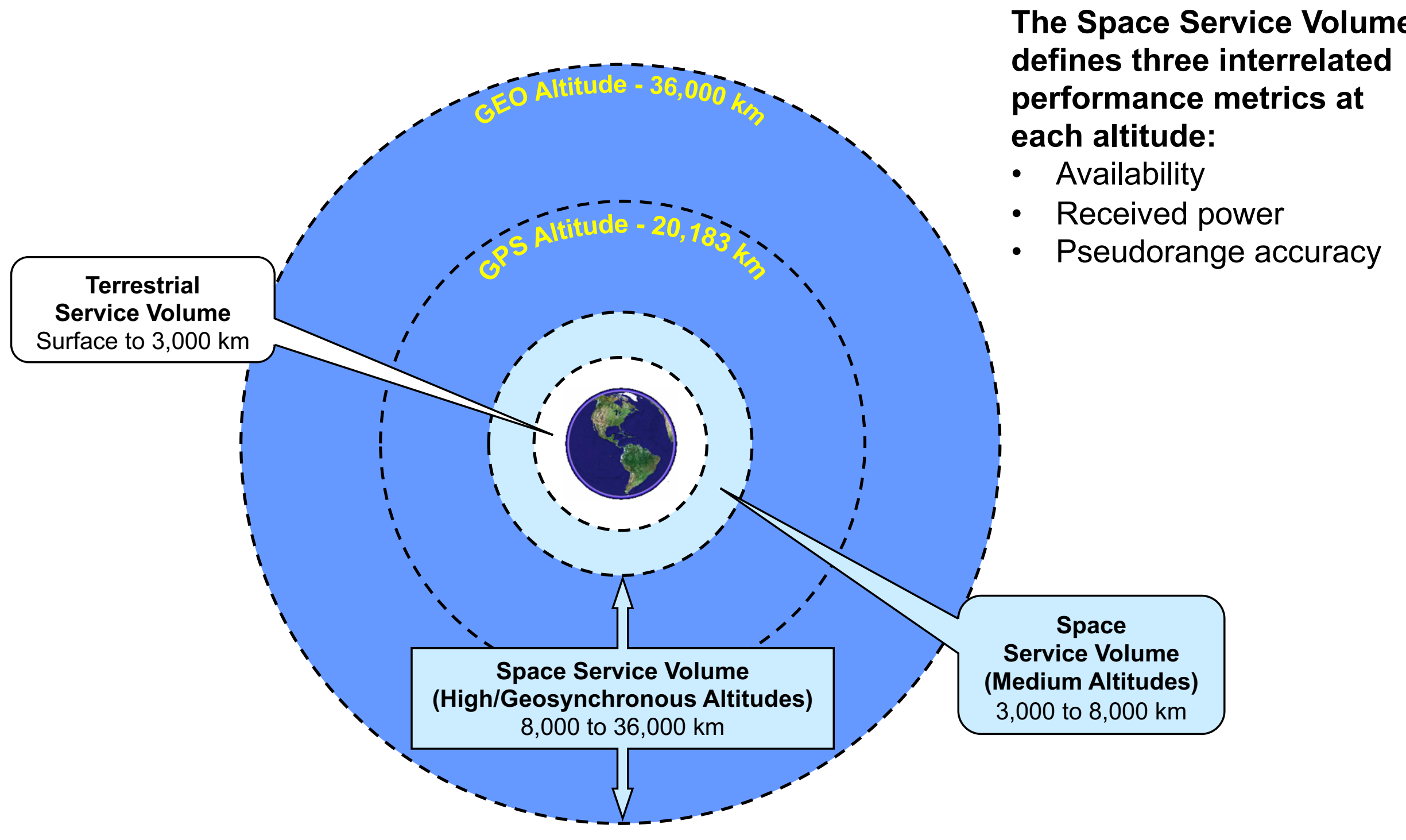




\section{GPS SSV}

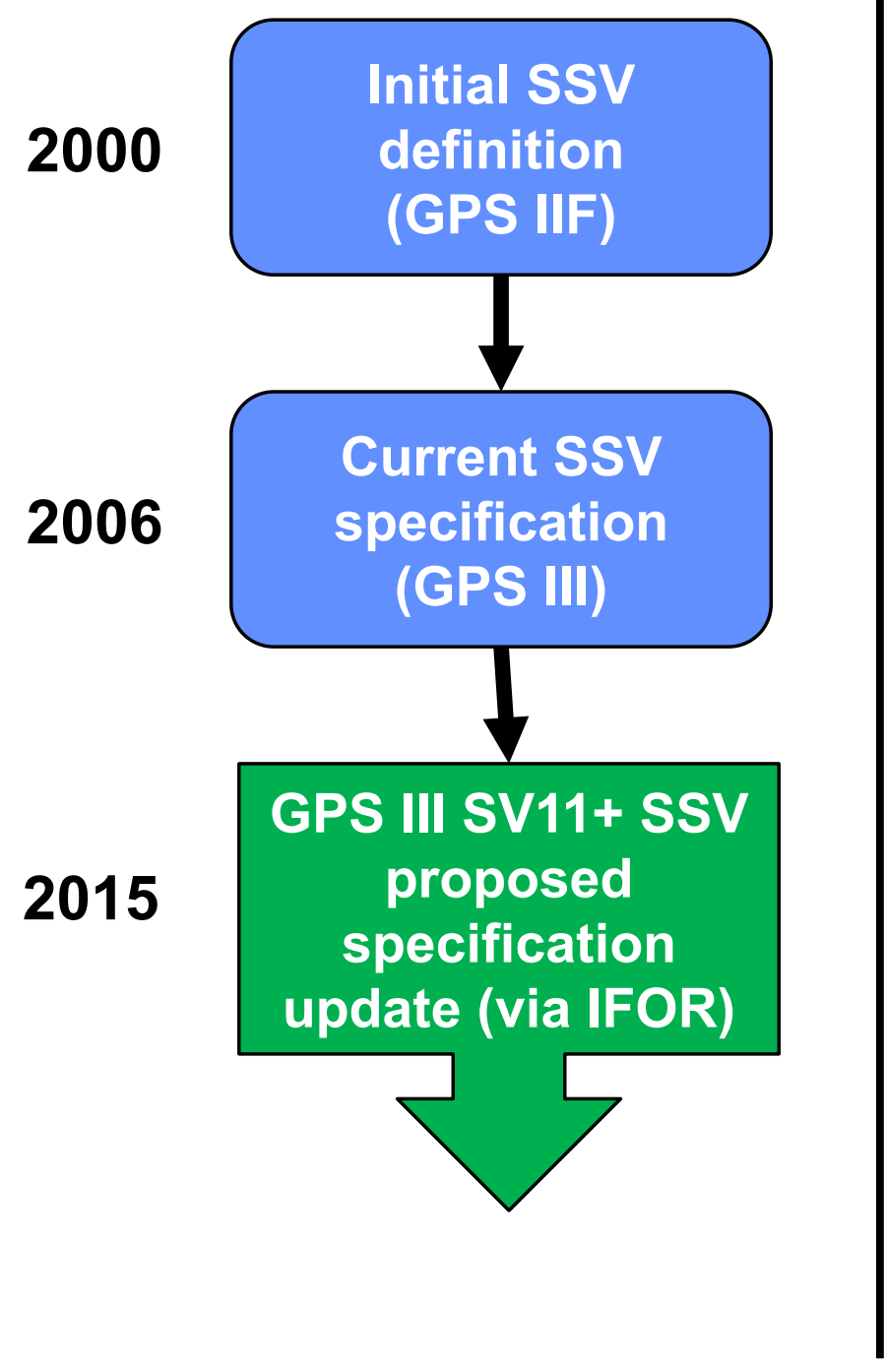

\section{Interoperable Multi-GNSS SSV}

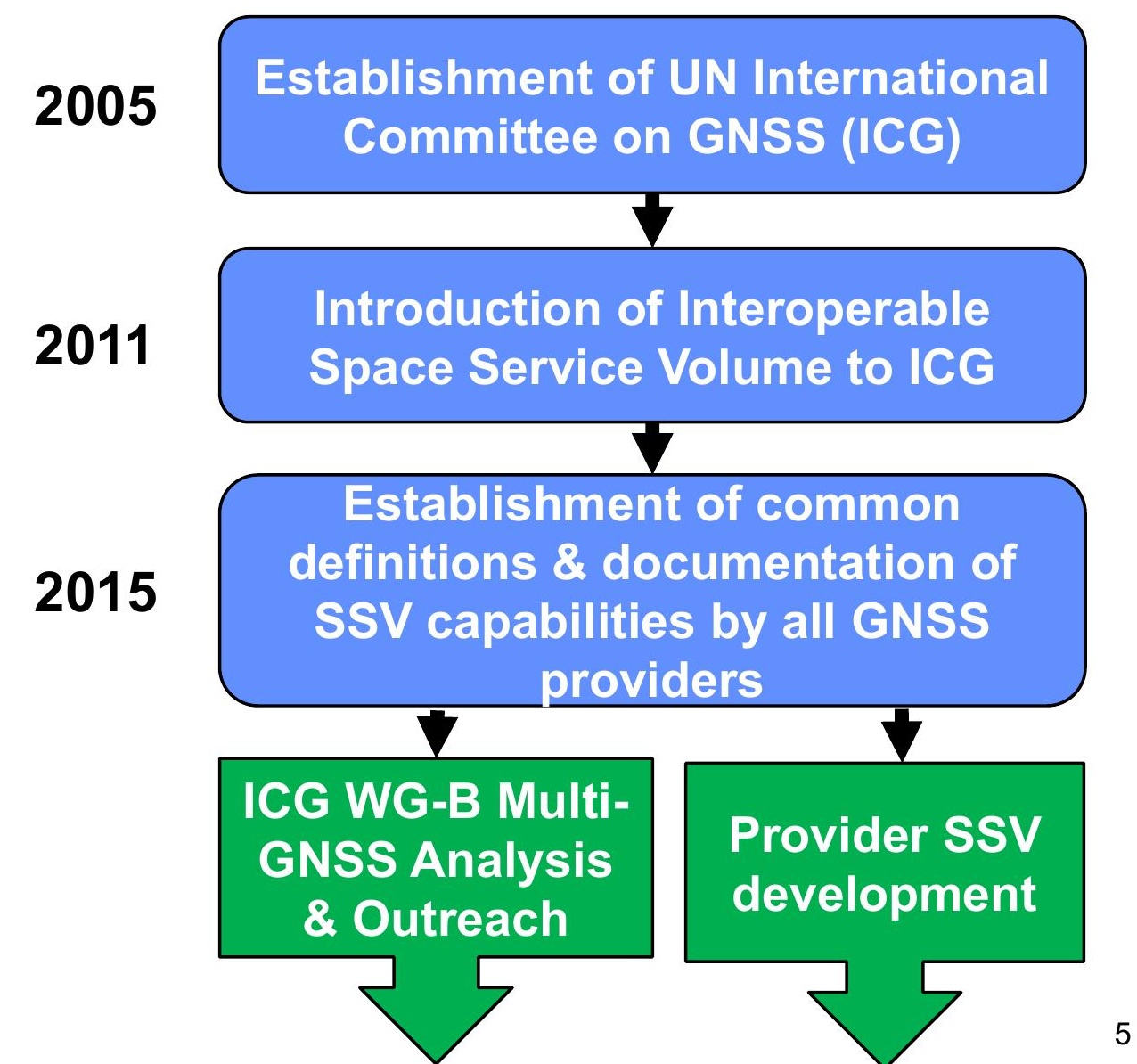


GPS SSV Progress 


\section{GPS Space Service Volume: Executive Summary}

- Current SSV specifications, developed with limited on-orbit knowledge, only capture performance provided by signals transmitted within $23.5^{\circ}$ (L1) or $26^{\circ}$ (L2/L5) of boresight.

- On-orbit data \& lessons learned since spec development show significant PNT performance improvements when the full aggregate signal is used.

- Numerous operational missions in High \& Geosynchronous Earth Orbit (HEO/GEO) utilize the full signal to enhance vehicle PNT performance

- Multiple stakeholders require this enhanced PNT performance to meet mission requirements.

- Failure to protect aggregate signal performance in future GPS designs creates the risk of significant loss of capability, and inability to further utilize performance for space users in HEO/GEO

- Protecting GPS aggregate signal performance ensures GPS preeminence in a developing multi-GNSS SSV environment

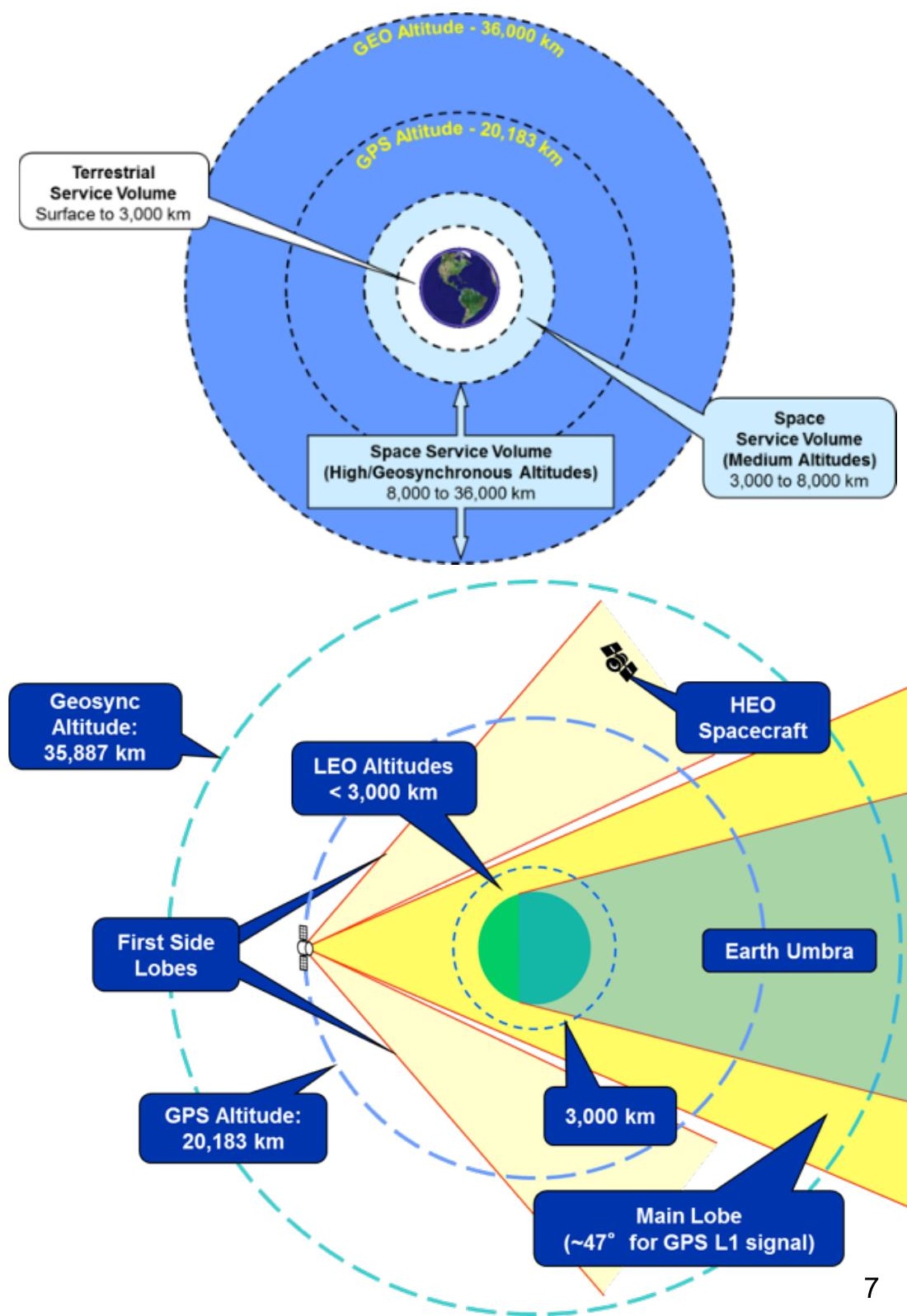




\section{The Promise of using GNSS for Real-Time Navigation in the Space Service Volume}

\section{Benefits of GNSS use in SSV:}

- Significantly improves real-time navigation performance (from: km-class to: meter-class)

- Supports quick trajectory maneuver recovery (from: 5-10 hours to: minutes)

- GNSS timing reduces need for expensive on-board clocks (from: $\$ 100$ sK $-\$ 1 \mathrm{M}$ to: $\$ 15 \mathrm{~K}-\$ 50 \mathrm{~K}$ )

- Supports increased satellite autonomy, lowering mission operations costs (savings up to $\$ 500-750 \mathrm{~K} / \mathrm{year}$ )

- Enables new/enhanced capabilities and better performance for HEO and GEO missions, such as:

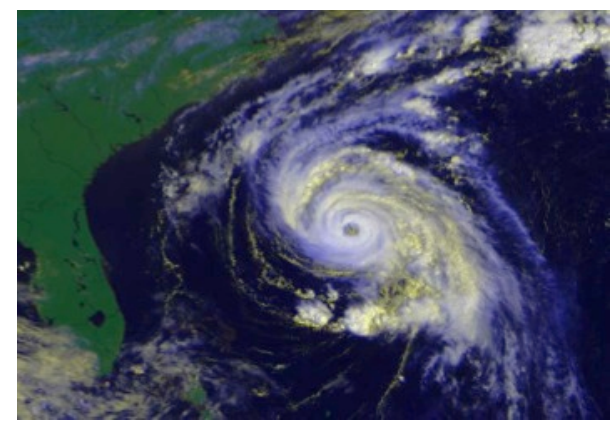

Earth Weather Prediction using Advanced Weather Satellites

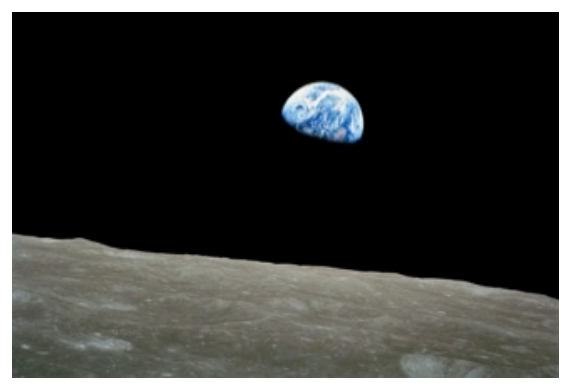

Launch Vehicle Upper Stages and Beyond-GEO applications

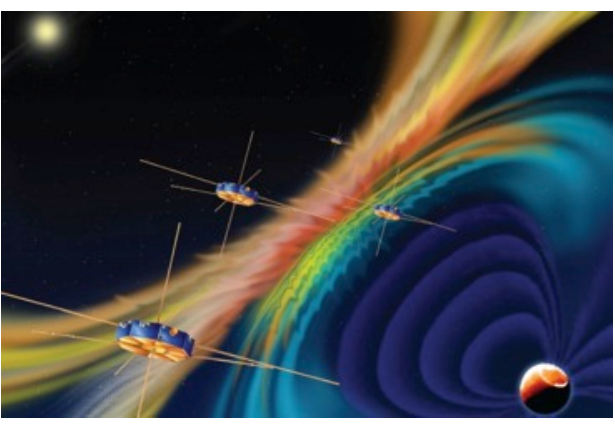

Space Weather Observations

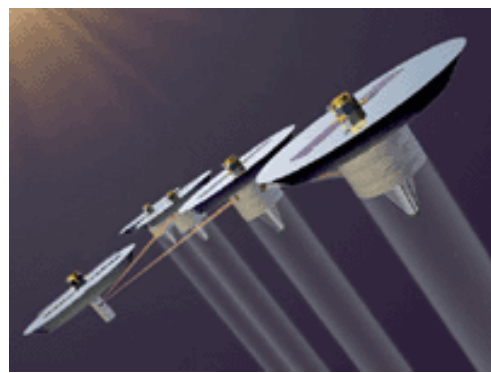

Formation Flying, Space Situational Awareness, Proximity Operations

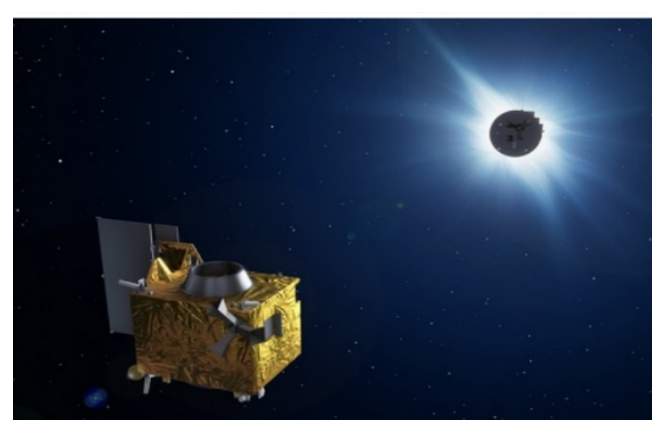

Precise Relative Positioning

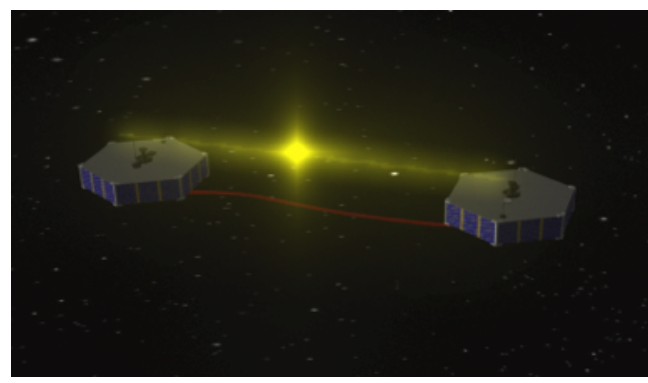

Precise Position Knowledge and Control at GEO 


\section{Key Civil Stakeholder: GOES-R}

- GOES-R, -S, -T, -U: $4^{\text {th }}$ generation NOAA operational weather satellites

- Launch: 19 Nov 2016, 15-year life

- Series operational through 2030s

- Driving requirements:

- Orbit position knowledge requirement (right)

- All performance requirements applicable through maneuvers, $<120 \mathrm{~min} / \mathrm{year}$ allowed exceedances

\begin{tabular}{|c|c|}
\hline Parameter & Requirement (m, 1-sigma) \\
\hline Radial & 33 \\
In-track & 25 \\
Cross-track & 25 \\
\hline
\end{tabular}

- Stringent navigation stability requirements

- Requirements unchanged for GOES-S, -T, -U

- GOES-R cannot meet stated mission requirements with SSV coverage as currently documented

- NASA-proposed requirement formulated as minimum-impact solution to meet GOES-R performance needs 


\section{GOES-R Mission Impact}

- Modeled each type of GOES-R maneuver at each GPS availability level

- Only 1 signal is necessary to recover nav performance; max outage is key metric

- At current required availability (red), post-maneuver errors exceed requirement in all cases, for up to 3 hours

- Proposed SSV requirement (blue) just bounds errors within GOES-R nav requirement

- RSS requirement is shown for illustration; in actuality, each component meets individually

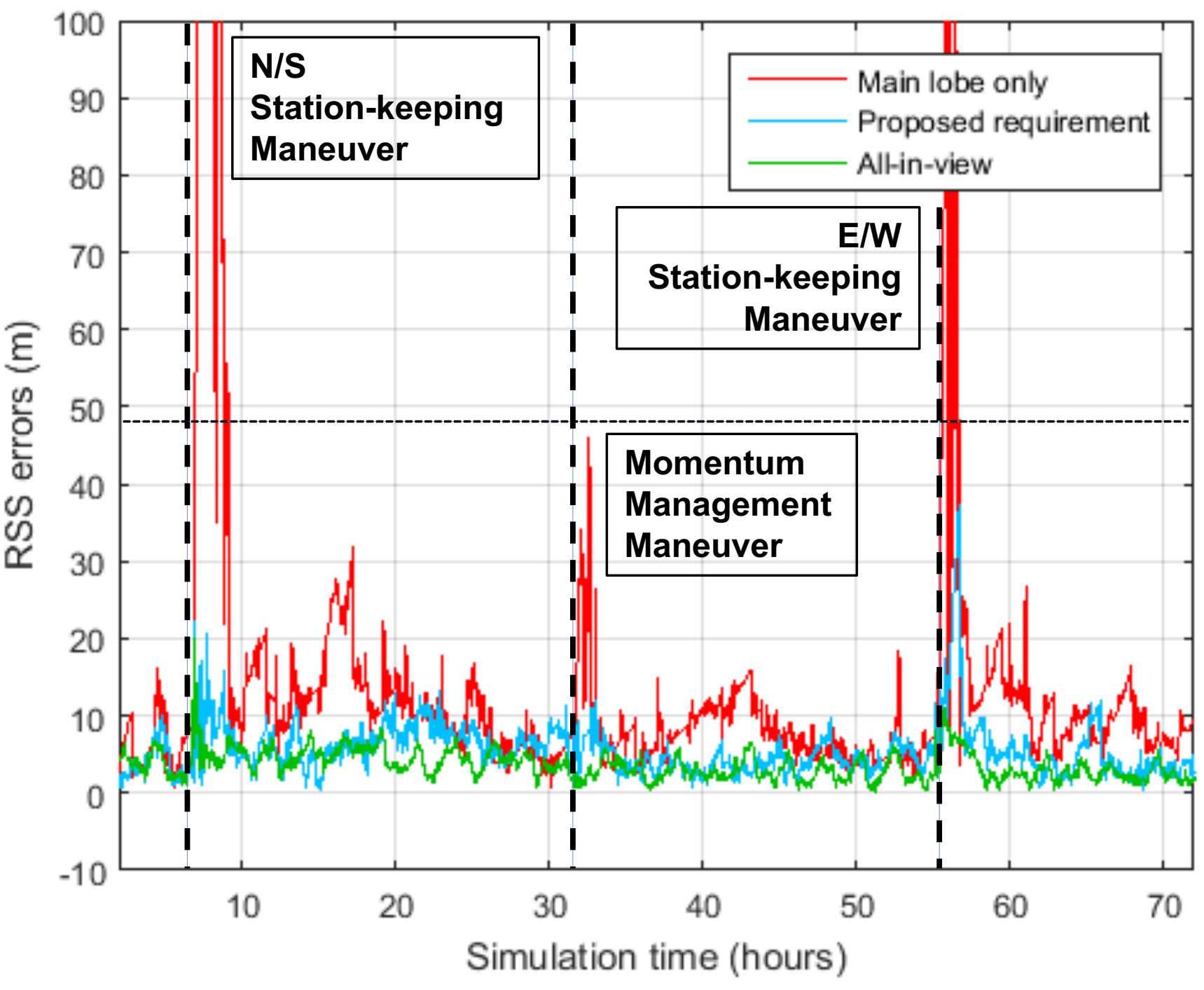

Errors with respect to simulation truth 


\section{Interagency Forum for Operational Requirements (IFOR) Current Status}

- Key participants:

- NASA, USAF (user side)

- SMC/GPV4 (GPS side)

- AFSPC/A5M (IFOR side)

- Original proposed recommendation from IFOR (Mar 2015):

1. Proceed with NASA requirement as objective requirement

2. SV11+ contractors to provide actual cost to meet objective

3. Users to confirm \& fund, based on actual cost

- Proposed recommendation after High Power Team (HPT) (Apr 2015):

- NASA/USAF to sign MOA for engagement throughout SV11+ acquisition

- Cost to be revisited at two milestones, based on additional insight from contractors

- NASA to coordinate civil funding for implementation, based on actual cost

- Current status:

- IFOR process has stalled; no progress since May

- MOA framework agreement reached, but staffing not initiated

- SV11+ Phase 1 is proceeding without stakeholder engagement or insight

- Phase 1 represents minimal-impact opportunity to implement proposed requirement for SV11+ series

- Independent Review Team established by AFSPC to advise on forward path 


\section{GPS SSV \\ Conclusions \& Way Forward}

- NASA has proposed an updated GPS SSV requirement to protect high-altitude space users from risk of reduced future GPS capability.

- Key civil example user is GOES-R

- Many other emerging users will require these capabilities in the future

- Available data suggests that the updated requirement can easily be met by a minimumperforming constellation of the previous design.

- If true, cost to implement would be documentation/ $\mathrm{V} \&$ only, not a hardware change

- But, in the absence of direct verification data, a risk remains that the requirement would not be met by the current and future designs

- This has led to a large gap between NASA and USAF impact estimates, with no mechanism to enforce technical transparency, coordination, or mitigations within IFOR.

- NASA seeks USAF engagement to seek and implement minimal-impact requirement based on best available data through SV11+ acquisition cycle

- Engagement has stalled at IFOR level - no progress on formal recommendation or MOA staffing

- $\quad$ NASA finds the proposed requirement critical to support future users in the SSV across the enterprise and is open to a commitment of funding based on a validated assessment.

- The proposed requirement is an innovative, whole-of-government approach that will protect and encourage next-generation capabilities in space at minimal cost.

- NASA encourages the work of the SSV Independent Review Team to provide independent analysis of proposed requirement and path forward. 


\section{Interoperable Multi-GNSS SSV Progress}




\section{International Committee on GNSS (ICG)}

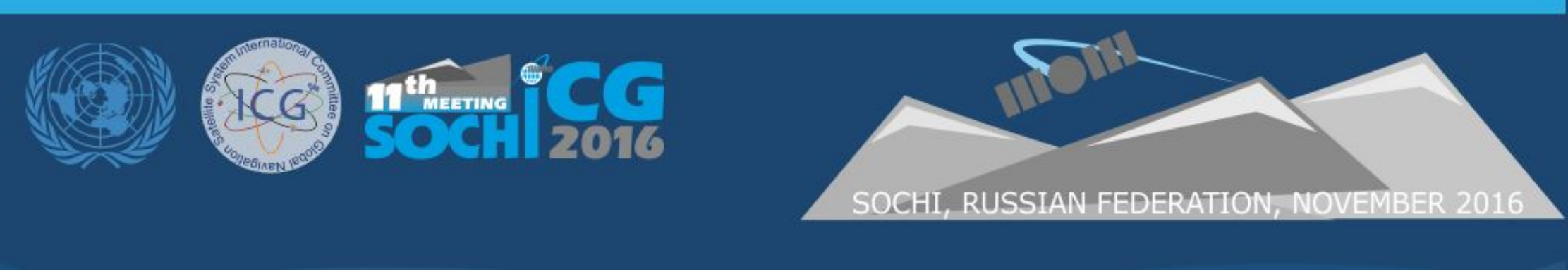

- Emerged from 3rd UN Conference on the Exploration and Peaceful Uses of Outer Space July 1999

- Promote the use of GNSS and its integration into infrastructures, particularly in developing countries

- Encourage compatibility \& interoperability among global and regional systems

- Members include:

- GNSS Providers: (U.S., EU, Russia, China, India, Japan)

- Other Member States of the United Nations

- International organizations/associations - Interagency Operations Advisory Group (IOAG) \& others

- $11^{\text {th }}$ annual meeting hosted by Russia in Sochi, November 6-11, 2016

http://www.oosa.unvienna.org/oosa/en/SAP/gnss/icg.html 


\section{Summary of ICG Multi-GNSS SSV Development Efforts To-Date}

- Interoperable, Multi-GNSS SSV coordination is accomplished as part of ICG Working Group B (WG-B): Enhancement of GNSS Performance, New Services and Capabilities

- ICG WG-B discussions have encouraged GPS, GLONASS, Galileo, BeiDou, QZSS, \& NAVIC to characterize performance for space users to GEO

- 2016 ICG meeting was held Nov. 6-11, in Sochi, Russia, where:

- All providers reaffirmed the criticality of GNSS for current and emerging space missions

- Participating members are finalizing a guidance booklet on GNSS SSV \& are jointly conducting analyses to characterize interoperability

- Stakeholder ICG members will coordinate a global outreach initiative to educate \& inform policy makers on the importance of a multi-GNSS SSV enabling space users to serve societal needs

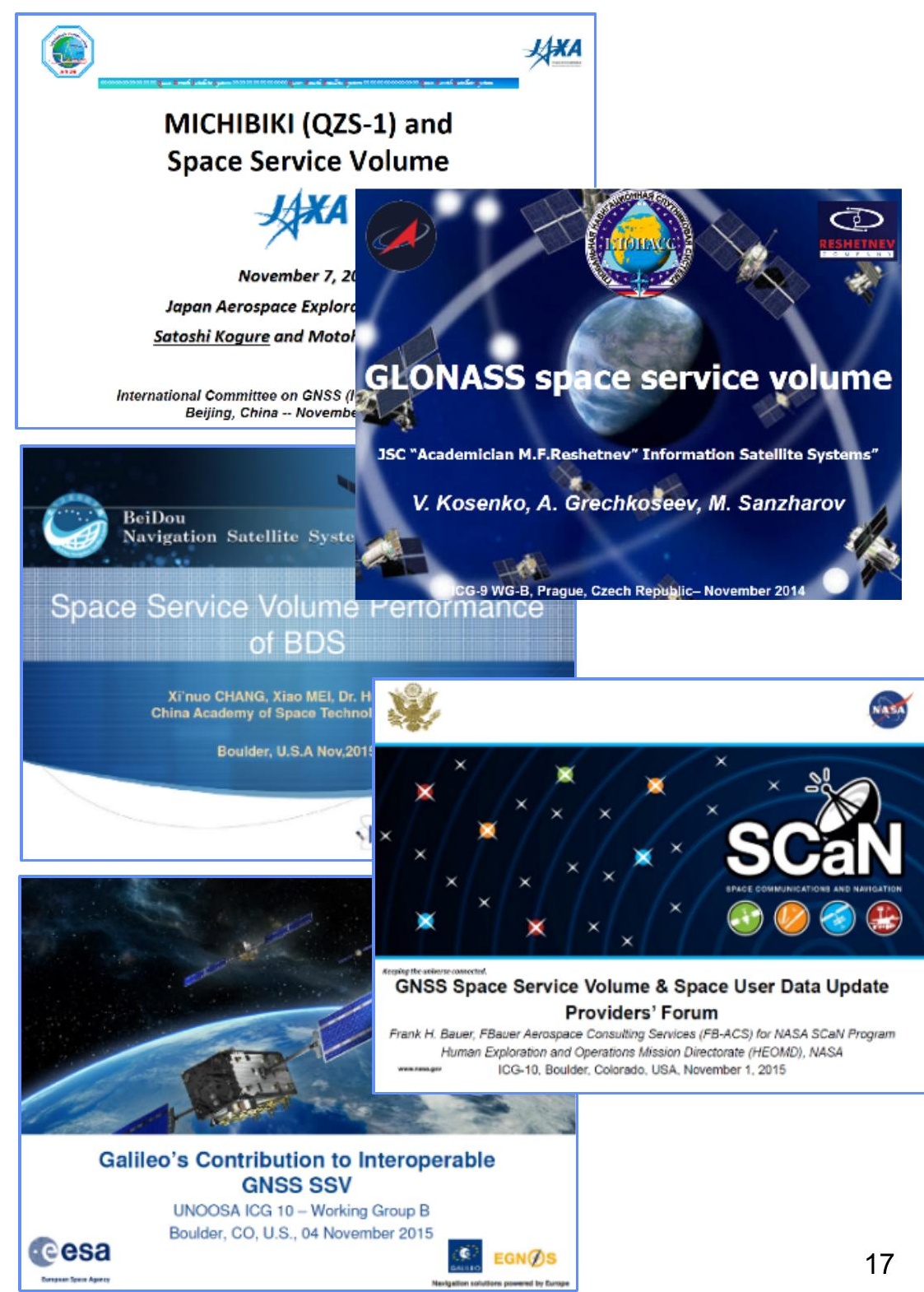




\section{ICG WG-B Joint SSV Analysis Effort}

- The ICG WG-B is performing an international analysis effort to demonstrate the benefits of an interoperable GNSS SSV, consisting of 3 phases of increasing complexity and fidelity:

- Phase 1 is a geometrical analysis of GNSS signal visibility at MEO \& GEO altitudes [completed May 2016]

- Phase 2 incorporates signal strength constraints to the geometrical analysis at GEO altitude [completed September 2016]

- Phase 3 extends Phase 2 to realistic user mission scenarios: GEO, HEO, and transLunar

- Phase 1 \& 2 Results were presented at the ICG-11 meeting Nov. 6-11 in Sochi, Russia

- Phase 3 mission planning kicked off and was discussed within ICG-11 WG B

- Analysis results will be captured in ICG SSV Booklet; joint int'l conference paper, journal articles, etc.

- Recently published in InsideGNSS, Nov/Dec 2016

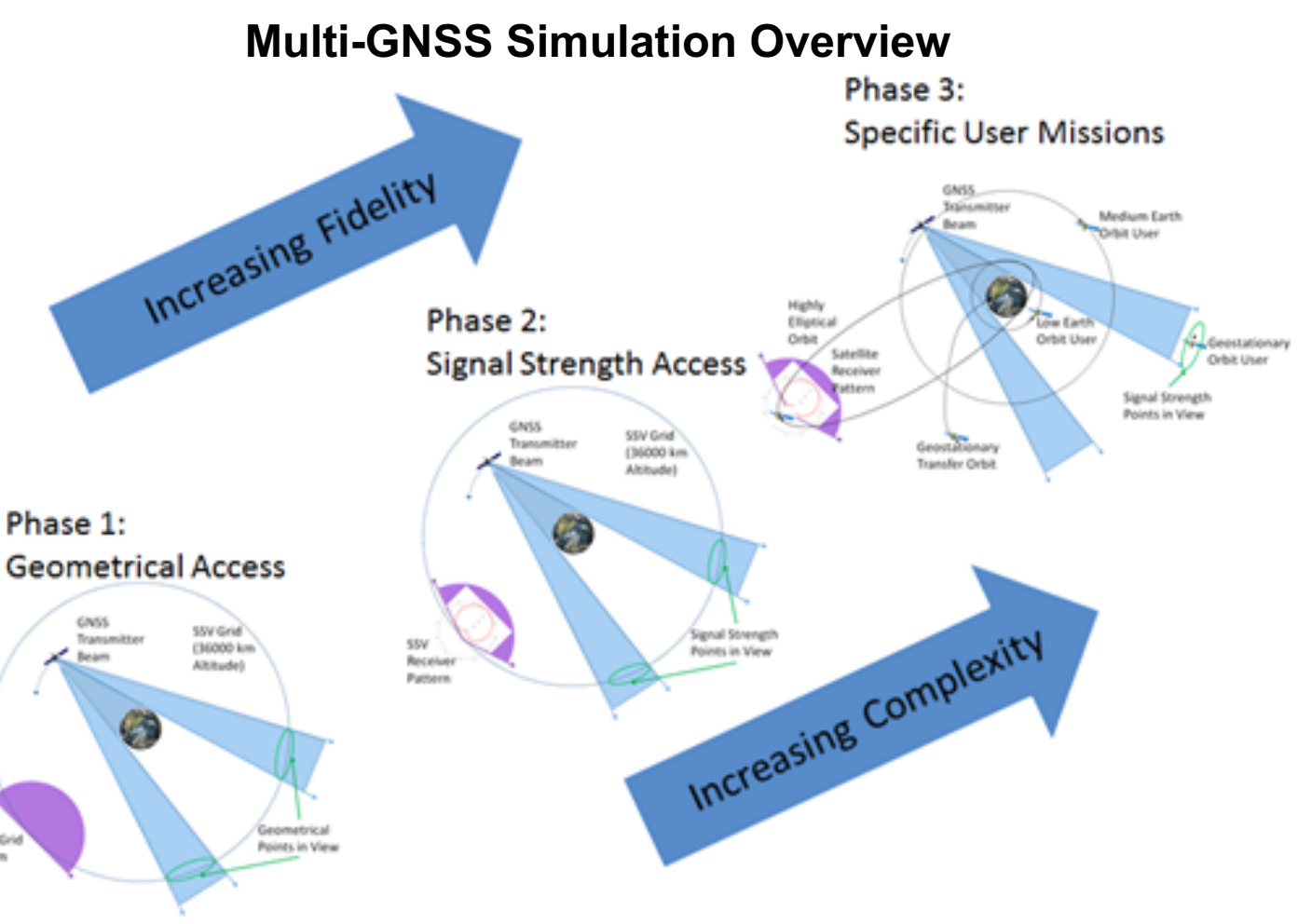

Multi-GNSS Simulation Video

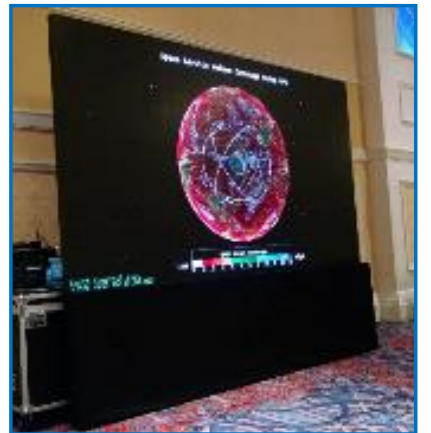


BeiDou

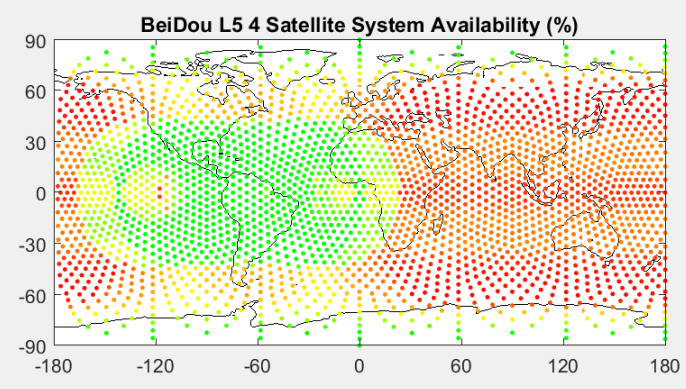

Average $45.4 \%$ availability GPS

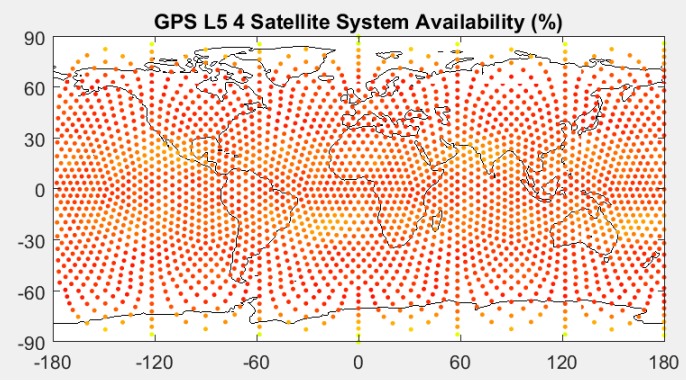

Average $15.6 \%$ availability
Galileo

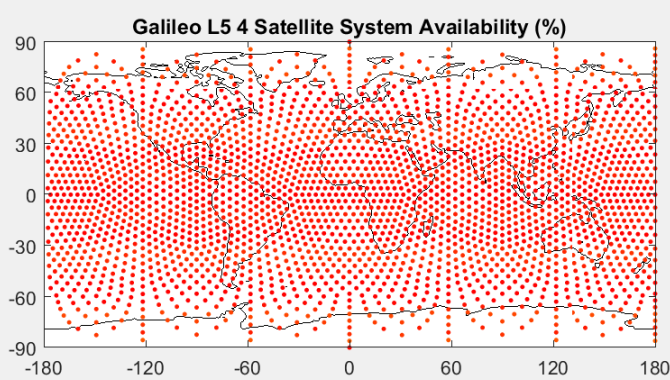

Average $4.2 \%$ availability

NAVIC

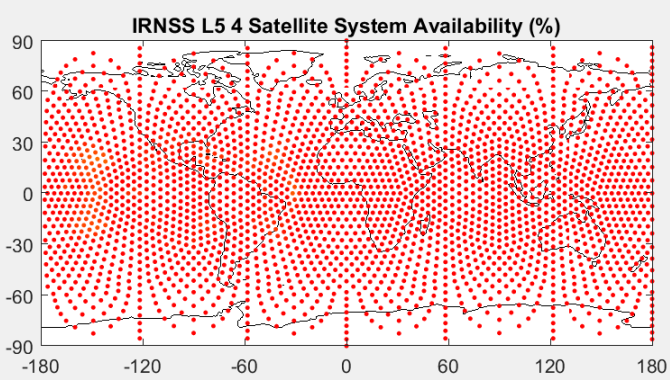

Average $0.6 \%$ availability
GLONASS

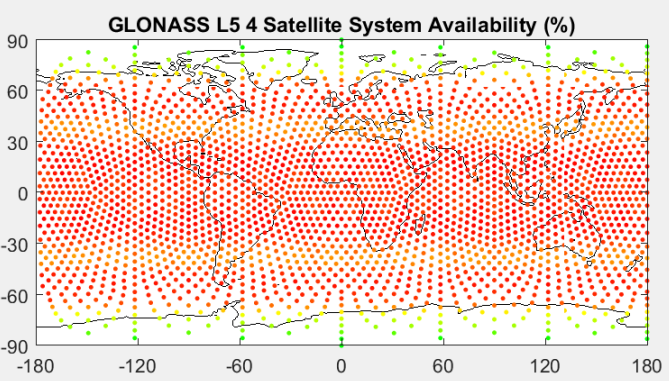

Average $14.5 \%$ availability

QZSS

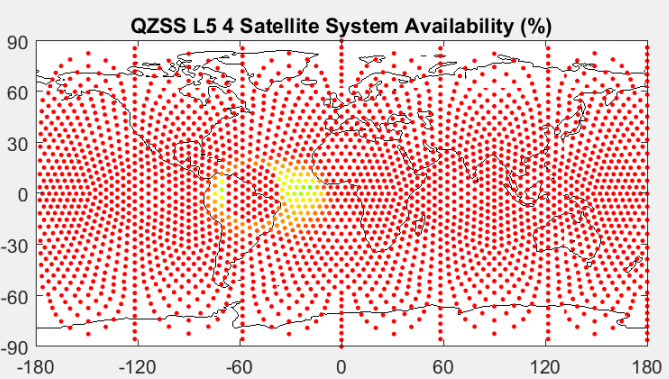

Average $1.5 \%$ availability
Interoperable GNSS achieves $100 \%$ system availability

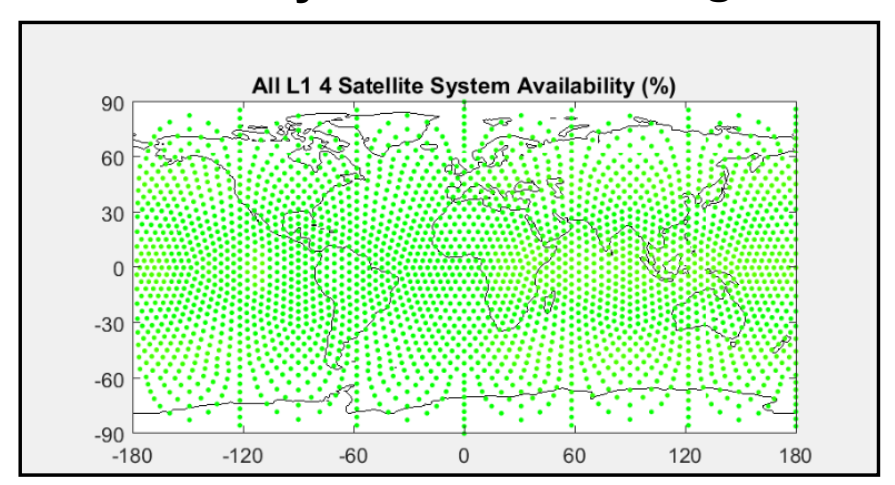




\section{ICG-11 SSV Recommendations}
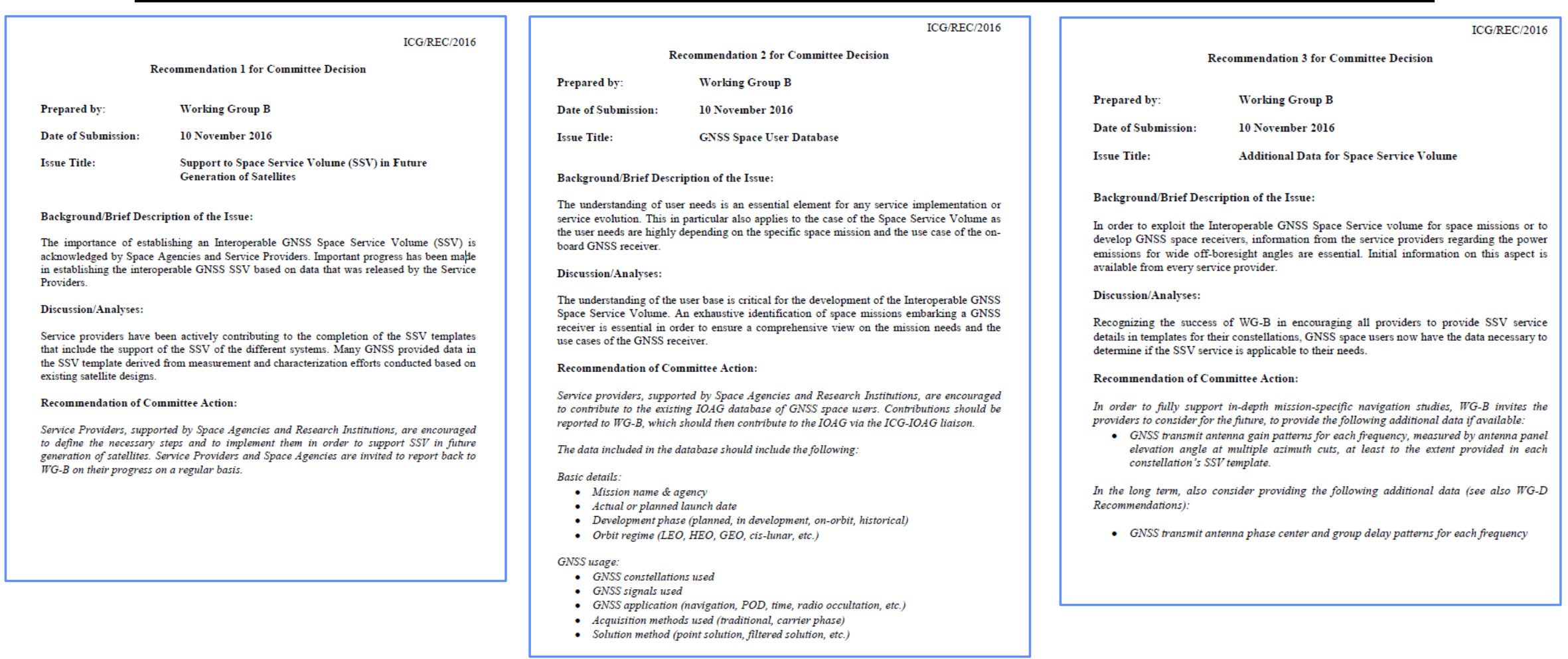

Service Providers, supported by Space Agencies \& Research Institutions encouraged to:

- Support SSV in future generation of satellites

- Contribute to GNSS space users database

- Measure and publish of GNSS antenna gain patterns to support SSV understanding \& use of aggregate signal 


\section{Conclusions}

- The Space Service Volume, first defined for GPS IIF in 2000, continues to evolve to meet high-altitude user needs.

- GPS led the way with a formal specification for GPSIII, requiring that GPS provides a core capability to space users.

- Today, we continue to work in parallel tracks to ensure that the SSV keeps pace with user demands.

- For GPS, with its well-characterized performance, we are working to update the SSV spec to capture the needs of emerging GPS-only users like GOES-R.

- In partnership with foreign GNSS providers, we are working jointly to characterize, analyze, document, and publish the capabilities of an interoperable multi-GNSS SSV with ultimate goal of provider specification.

- Both approaches are equally critical: a robust GPS capability will enable and enhance new missions in single-system applications, while an interoperable GNSS SSV ensures that a wider capability is available as needed. 


\section{Backup Charts}




\section{Before We Begin...}

- Oct 20, 2016: Guinness World Record awarded to NASA's

Magnetospheric MultiScale (MMS) mission for the highestaltitude GPS fix ever recorded: 70,135 km (2x geostationary altitude)

- Feb 2017: MMS apogee raise to $160,000 \mathrm{~km}$

- New record to follow?

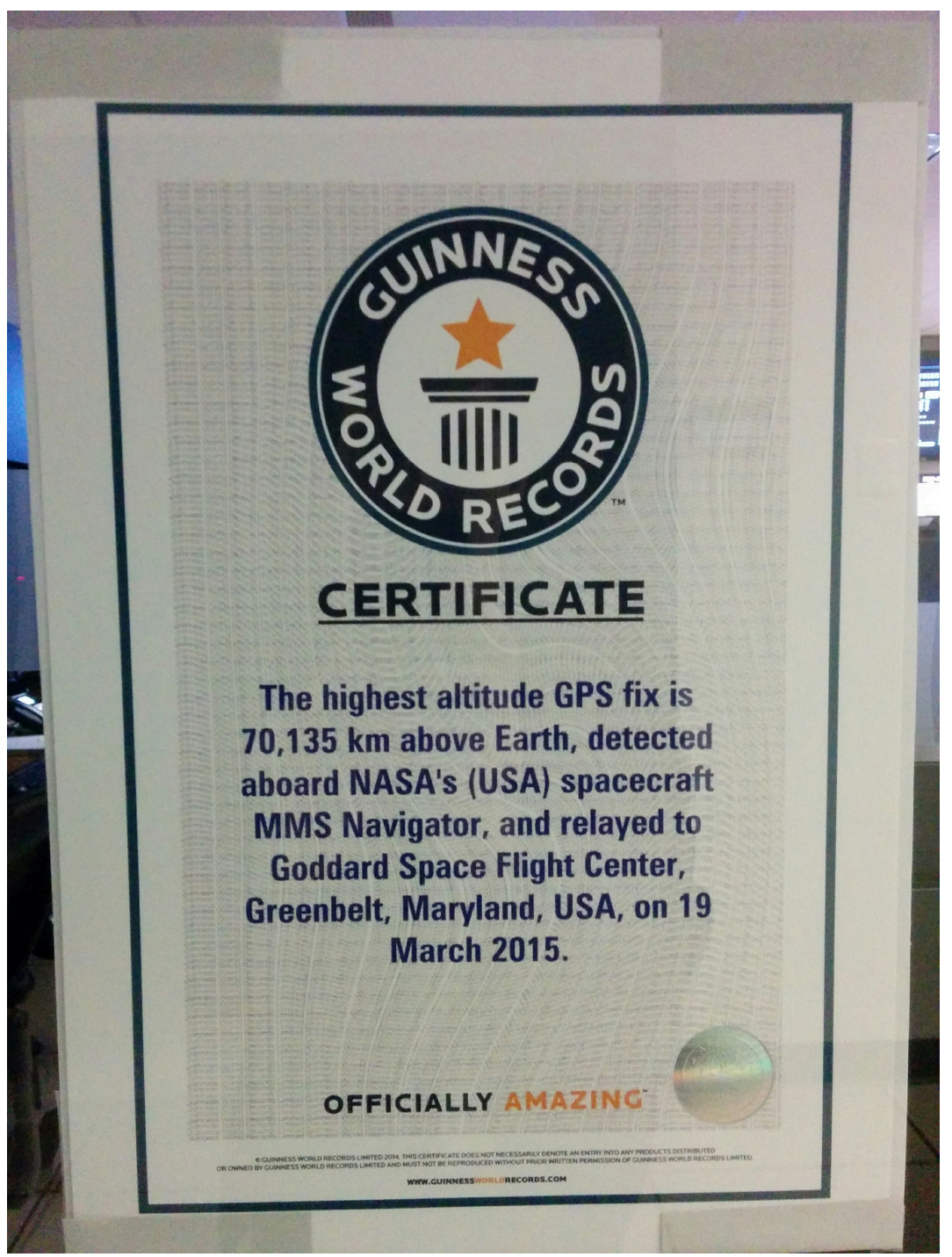

